\title{
Preparation, characterization and optimization of cross-linked fructosyltransferase aggregates for the production of prebiotic fructooligosaccharides
}

\author{
Adedeji N. Ademakinwa ${ }^{1,2}$, Zainab A. Ayinla ${ }^{2}$, Ofelia G. Omitogun ${ }^{2}$, Femi K. Agboola ${ }^{2 *}$ \\ ${ }^{1}$ Elizade University, Ilara-Mokin, Ondo State, Nigeria \\ ${ }^{2}$ Obafemi Awolowo University, Ile-Ife, Nigeria
}

\begin{abstract}
In this study, the preparation, characterization, and evaluation of the cross-linked enzyme aggregates (CLEAs) of fructosyltransferase isolated from the mutant-type Aureobasidium pullulans NAC8 for the production of fructooligosaccharides (FOS) with prebiotic properties were investigated. The reaction conditions were optimized statistically by using response surface methodology. CLEAs were characterized by using both photo-microscopy and scanning electron microscopy energy-dispersive X-ray spectroscopy (SEM-EDX). The secondary structure of the protein was predicted by using Fourier transform infrared spectroscopy (FTIR). FOSs produced as a result of the biotransformation of sucrose was quantified by using a chemometric analysis of FTIR spectral data. The prepared FOSs were investigated for the prebiotic effects under anaerobic conditions using a standard strain of Lactobacillus sp. Following were the statistically determined, optimum conditions for the production of FOSs: $5 \%$ (v/v) glutaraldehyde, $\mathrm{pH} 5.5$, and a temperature of $32^{\circ} \mathrm{C}$. The values of $R^{2}, F$ value, adjusted precision, and coefficient of variation were found to be $0.94,11.50,9.34$, and $35.34 \%$, respectively. Validation of the model resulted in the reusability of the CLEAs in six reaction cycles as it retained $70 \%$ of the residual activity. The SEM-EDX and photomicrographic analysis showed that the sizes of CLEAs varied, which ranged between 10 and $40 \mu \mathrm{m}$. Aggregation leads to the formation of a higher number of beta sheets in the CLEAs than that in the free enzyme. The total FOSs produced by the CLEAs was around $57 \%(\mathrm{w} / \mathrm{v})$. A. pullulans FOS supplemented to the medium had a stimulatory effect on the growth of bi dobacteria compared with the control medium. The growth curves of Lactobacillus sp. reached the plateau phase after $24 \mathrm{~h}$ of cultivation with FOS. The CLEA of fructosyltransferase catalyzed the biocatalysis of sucrose to FOSs, a process with a biotechnological importance as prebiotics.
\end{abstract}

Key words: Aureobasidium pullulans NAC8, cross-linked enzyme aggregates (CLEAs), fructosyltransferase, fructooligosaccharides, statistical optimization, SEM-EDX

\section{Introduction}

Fructosyltransferases (EC 2.4.1.9) are enzymes that catalyze the transfer of fructose units from a sucrose molecule to another molecule of sucrose or to fructose in a process that yields higher amounts of fructooligosaccharide (FOS) units (Maiorano et al., 2009). FOSs have also been termed as fructose oligomers; they have a terminal glucose residue that is linked to fructose via glycosidic bond $(\beta-2,1)$. FOSs are nondigestible carbohydrates that represent one of the major classes of bifidogenic oligosaccharides; they have/show important health benefits as prebiotics (Sabater-Molina et al.,
2009). Research in industrial enzymology has resulted in the large-scale production of FOSs by the method of enzymatic transformation of fructosyltransferases and or $\beta$-fructofuranosidases on sucrose (Flores-Maltos et al., 2014). Industrially, FOS production can be classified under two broad categories: the batch system, where the free enzyme is used and a continuous system, in which immobilized cells and or enzymes are used. Enzyme immobilization, mostly in bioreactors, offers a great potential due to the possibility of the high-enzyme load. This leads to high volumetric productivities and a finite control on the extension of the reaction itself and a simpli-

*Corresponding author: Obafemi Awolowo University, Ile-Ife, Nigeria; e-mail: fkagbo@oauife.edu.ng 
fication of the downstream process. Furthermore, enzyme immobilization offers the possibility of recovering and reusing the biocatalyst (Fernandez et al., 2005). It offers numerous advantages such as the prevention of degradation of enzyme and substrate inhibition, which often is seen with free enzymes. In addition, it will be more useful if the process of immobilization is automated (Sheldon, 2007; Fernandes, 2010).

Traditionally, the technique of enzyme immobilization is performed using a myriad of methods, including entrapment, binding to a solid carrier, and micro-encapsulation; these methods are referred to as the carriertype immobilization. The carrier-type immobilization technique inevitably leads to the dilution of the catalytic activity resulting from the introduction of a large proportion of noncatalytic mass. This results in a lower volumetric yield and a lower productivity of the catalyst (Cao et al., 2000). The other types of immobilization, where there is no need to bind to a carrier molecule, are broadly termed as CLEAs. Immobilization via the crosslinking of enzyme molecules with a bifunctional crosslinking agent is a carrier-free method and the resulting biocatalyst essentially comprises $100 \%$ active enzyme. There are several cross-linking agents that can cross-link proteins and enzymes, such as glutaraldehyde, dextran polyaldehyde, diepoxide, $p$-benzoquinone, L-lysine, pectin, and polyethyethyleneimines. Among these, glutaraldehyde is the most frequently used cross-linking agent (Cao et al., 2003; Mateo et al., 2007; Wang et al., 2011; Ayhan et al., 2012; Talekar et al., 2014); however, it has some drawbacks. For example, glutaraldehyde has been shown to disrupt the activity of nitrilase as it can easily penetrate the enzyme due to its small size $(0.1 \mathrm{kDa})$ (Valdes et al., 2001). In general, the mechanism of action of the aforementioned cross-linking agents to form CLEA is based on the interaction between the protein's amino groups and the reactive groups of the cross-linker resulting in the formation of covalent bonds (Schoevaart et al., 2004). Glutaraldehyde is the most common crosslinker; it interacts with the aggregated proteins (aggregation occurs by precipitating the protein using ammonium sulfate, or other organic solvents).

CLEAs are obtained by precipitating nonpurified enzymes to form solid particles that bind with each other via noncovalent bonds (Tischer and Kasche et al., 1999). They become permanently insoluble after they covalently bind with a cross-linking agent such as glutar- aldehyde. In a typical reaction model, CLEAs exhibit greater activity (more than 10-fold) than their corresponding biocatalysts that are immobilized using a carrier. Comparatively, glutaraldehyde has a negligible molecular weight compared to the protein of interest which is to be immobilized; therefore, the resulting immobilized biocatalyst is nearly $100 \%$ target protein of interest to be crosslinked with the assumption that the weight of glutaraldehyde is negligible. In addition, CLEAs are insoluble and hence can be easily recovered from the reaction milieu by performing low-speed centrifugation (Park et al., 2010). In addition, CLEAs offer an avenue where the proteins' catalytic features combine readily with the mechanical behavior of an industrially used biocatalyst.

The advantages offered by the immobilization of FOSproducing enzyme using CLEAs compared to the free enzyme are numerous, such as $\mathrm{pH}$ and thermal stability, easy separation of products from biocatalysts, and a condition in which a continuous process can be developed. Overall, an increased operational stability is envisaged. For the large-scale production of FOS, the FOS-producing enzymes have been immobilized on porous glass, porous silica (Hayashi et al., 1993), ion-exchange resins (Yun and Song, 1996), gluten (Chien et al., 2001), polymethacrylate (Ghazi et al., 2005), macroporous beads (Tanriseven and Aslan, 2005), and calcium alginate (Jung et al., 2011).

To the best of our knowledge, the use of fructosyltransferase CLEAs in the synthesis and large-scale production of FOSs has not been fully exploited. The only recent report on the synthesis of FOSs involved the use of levansucrase from Bacillus subtilis (Ortiz-Soto et al., 2009). This implies that the possibility of the synthesis of FOSs by using carrier-free immobilized fructosyltransferase is worth investigating.

Therefore, in this study, we synthesized cross-linked fructosyltransferase aggregates from an improved mutant strain of Aureobasidium pullulans NAC8 (Ademakinwa and Agboola, 2016; Ademakinwa et al., 2017), and we developed a mathematical model for the industrial production by optimizing the scale-up conditions using statistical techniques. The characterization of CLEAs was performed using photomicroscope and scanning electron microscopy energy-dispersive X-ray spectroscopy (SEM-EDX). The structural changes of CLEAs after cross-linking with glutaraldehyde were investigated by way of a peak fitting analysis of the amide I band of 
the spectral data obtained by Fourier transform infrared spectroscopy (FTIR). The reusability of (the) CLEAs was also investigated over several reaction cycles. The reaction products of the CLEA biocatalysis of sucrose were purified using activated charcoal, and the sugar content was quantified using multivariate data analysis of the FTIR spectral data. The use of FOSs as prebiotics was investigated under anaerobic conditions using Lactobacillus sp. as the probiotic strain of choice.

\section{Materials and methods}

\section{Reagents}

Glutaraldehyde was obtained from Fluka, Germany. Sucrose, activated charcoal, potassium bromide, bovine serum albumin, glucose, sucrose, and fructose were obtained from Sigma Aldrich, USA. The commercial FOS standard was purchased from Source Naturals, Santa Cruz, California, USA, and it was purified on activated charcoal (Sigma Aldrich, USA) prior to use. All other reagents were of analytical grade and used as provided without further purification.

\section{Microorganisms and maintenance}

The fungus, Aureobasidium pullulans, used in the study was genetically modified using chemical mutagenesis of the wild-type Aureobasidium pullulans NAC8 strain (Accession no. KX023301) (Ademakinwa and Agboola, 2016; Ademakinwa et al., 2017). The fungus was maintained on the malt extract agar slant at $4^{\circ} \mathrm{C}$ and was routinely subcultured after every 7 days.

\section{Submerged fermentation and isolation of intracellular fructosyltransferase}

The medium for enzyme production contained (w/v) the following ingredients: sucrose (20\%), yeast extract (0.5\%), $\mathrm{NaNO}_{3}(1 \%), \mathrm{MgSO}_{4} \cdot 7 \mathrm{H}_{2} \mathrm{O}(0.05 \%), \mathrm{KH}_{2} \mathrm{PO}_{4}$ $(0.25 \%), \mathrm{NH}_{4} \mathrm{Cl}(0.5 \%)$, and $\mathrm{NaCl}(0.25 \%)$ with an initial $\mathrm{pH}$ 5.5. After $72 \mathrm{~h}$ of incubation, the cultured broth was centrifuged at $6000 \times g$ for $10 \mathrm{~min}$ at $4{ }^{\circ} \mathrm{C}$ using a refrigerated centrifuge. The pellet served as the source of mycelial fructosyltransferase. The mycelia were washed with several rounds of distilled water and blotted to dryness. The intracellular FOS-producing enzymes in the mycelia of the improved mutant strain of $A$. pullulans were extracted by grinding the mycelia with acid washed sand $(\mathrm{pH}$ 7.0) $(1: 1)$ in a clean prechilled mortar using $0.1 \mathrm{M}$ acetate buffer, $\mathrm{pH}$ 5.0. The material was homo- genized at room temperature. The so-obtained homogenate was centrifuged at $4000 \times g$ for $20 \mathrm{~min}$, and the resulting supernatant was used as the source of intracellular enzyme. All operations were performed on the ice at $4{ }^{\circ} \mathrm{C}$ (Ademakinwa et al., 2017).

\section{Fructosyltransferase assay}

Fructosyltransferase activity was determined by incubating an aliquot of the enzyme solution $(0.1 \mathrm{ml})$ with $0.9 \mathrm{ml}$ of $60 \%$ sucrose $(\mathrm{w} / \mathrm{v})$ in $0.1 \mathrm{M}$ acetate buffer (pH 5.5) at $45^{\circ} \mathrm{C}$ for $60 \mathrm{~min}$ in a water bath. The reaction was stopped by boiling the mixture at $100^{\circ} \mathrm{C}$ for $20 \mathrm{~min}$. The released glucose was quantified using a glucose oxidase-peroxidase (GOD-POD) kit (Sigma), using a spectrophotometer at $505 \mathrm{~nm}$. One unit of fructosyltransferase was defined as the amount of enzyme activity required to produce $1 \mu \mathrm{mol}$ of glucose per minute under the described conditions (Ganaie et al., 2014, Ademakinwa et al., 2017).

\section{Synthesis of CLEAs}

The optimization of cross-linking reactions was conducted statistically using the response surface methodology (RSM) model (Box-Behnken design (BBD)). Using one factor at a time (OFAT), the determination of the best precipitant from toluene, acetone, ethanol, and ammonium sulfate, at concentrations ranging between 40 and $70 \%$ (v/v) in $0.2 \mathrm{M}$ acetate buffer, $\mathrm{pH} 5.5$ was performed (but not included in this report) (Ortiz-Soto et al., 2009). The effect of cross-linking with glutaraldehyde was explored in a concentration ranging between 1 and 20\% (v/v). After cross-linking, the mixture was quenched with $900 \mu \mathrm{l}$ of $0.2 \mathrm{M}$ acetate buffer, $\mathrm{pH}$ 5.5. The quenched samples were assayed for enzymatic activity. An aliquot from the suspension, which contained both CLEAs and a residualfree enzyme, was assayed for activity. The activity of the free enzyme fraction was assessed on a sample after removal of CLEAs by centrifugation (Ortiz-Soto et al., 2009). The difference in activities between the two samples was the actual CLEA activity. This approach is the most accurate way of determining the activity of CLEAs, without having to wash and redisperse, which can increase clotting of the CLEA and thereby cause limitations in mass-transport (Schooevart et al., 2004). Reactions using sucrose, both as a donor and as an acceptor, were performed using a medium containing $600 \mathrm{~g} / 1$ of sucrose (pH 6.0), $50 \mathrm{mM}$ sodium phosphate 
Table 1. Selected variables and their assigned levels by Box-Behnken design (BBD)

\begin{tabular}{c|c|c|c|c|c|c}
\hline \multirow{2}{*}{ Variables } & \multirow{2}{*}{ Component } & \multirow{2}{*}{ Unit } & \multirow{2}{*}{ Range } & \multicolumn{3}{|c}{ Levels of variables studied } \\
\cline { 5 - 7 } & & & & -1 & 0 & +1 \\
\hline $\mathrm{A}$ & $\mathrm{pH}$ & - & $4-6$ & 4 & 5.5 & 6 \\
\hline $\mathrm{B}$ & temperature & ${ }^{\circ} \mathrm{C}$ & $4-60$ & 4 & 32 & 60 \\
\hline $\mathrm{C}$ & glutaraldehyde & $\%(\mathrm{v} / \mathrm{v})$ & $1-10$ & 1 & 5.0 & 10 \\
\hline
\end{tabular}

at $37^{\circ} \mathrm{C}$. Preparation of CLEA, as well as the enzyme assays, were performed at least thrice.

\section{Aggregation yield}

The aggregation yield is defined as the activity recovered in the CLEAs when compared with the total activity of the soluble enzyme in the experiment (OrtizSoto et al., 2009).

\section{Statistical optimization for cross-linked fructosyltransferase aggregates}

The choice of precipitants (toluene, acetone, and ammonium sulfate), as well as their concentration leading to the formation of fructosyltransferase CLEA, was determined using the OFAT approach. Statistical optimization was performed using the BBD and several factors influencing the formation of CLEA as well as the catalysis, namely, the concentration of cross-linker, temperature, and $\mathrm{pH}$, were investigated.

\section{RSM-based optimization}

In this study, the concentration of glutaraldehyde and the optimum $\mathrm{pH}$ and temperature were studied at three different levels $(-1,0$, and +1$)$ (Table 1$)$. The levels were termed as $+1,0$, and -1 which indicates high, central (middle), and low variables, respectively. The variables were set at a central coded value of zero. The minimum and maximum ranges of variables were obtained based on previously conducted independent experiments (data not shown). For each experimental run, the fructosyltransferase activity was measured in triplicate. The aggregation yield was analyzed using a second-order polynomial equation. A multiple regression procedure was used to fit the data into the equation. For the correlation of the relationship that exists between the response (aggregation yield) and the independent variables, the second-order polynomial function was thus fitted. This was used to predict the optimal point. Following is the equation for three factors:

$$
\begin{aligned}
X & =\beta_{0}+\beta_{1} Y_{1}+\beta_{2} Y_{2}+\beta_{3} Y_{3}+\beta_{12} Y_{1} Y_{2}+ \\
& +\beta_{13} Y_{1} Y_{3}+\beta_{23} Y_{2} Y_{3}+\beta_{11} Y_{12}+\beta_{22} Y_{22}+\beta_{33} Y_{32}
\end{aligned}
$$

where $X$ is the predicted response; $\beta_{0}$ is the model constant; $Y_{1}, Y_{2}$, and $Y_{3}$ are independent variables; $\beta_{1}, \beta_{2}$, and $\beta_{3}$ are linear coefficients; $\beta_{12}, \beta_{13}$, and $\beta_{23}$ are cross product coefficients; and $\beta_{11}, \beta_{22}$, and $\beta_{33}$ are the quadratic coefficients. The model equation for the analysis is given as representing the constant process effect in total, the linear $\left(Y_{\mathrm{i}}\right)$, quadratic effect $\left(Y_{\mathrm{j}}\right)$, and the effect of interaction between $Y_{\mathrm{i}}$ and $Y_{\mathrm{j}}$ for the aggregation yield (Haaland, 1989; Ademakinwa et al., 2017).

\section{Model validation}

The model validation for the catalysis by CLEA was performed by preparing the CLEAs using the optimum values $\left(5 \%(\mathrm{v} / \mathrm{v})\right.$ glutaraldehyde, $\mathrm{pH} 5.5$, and $\left.32^{\circ} \mathrm{C}\right)$ predicted by the BBD RSM model. The experimental, as well as the predicted values for the aggregation yield, were confirmed.

\section{Statistical analysis}

The RSM model, as well as the analysis of variance (ANOVA), were designed using Design Expert 7.1.5. Positive effects in terms of aggregation yield were considered significant for $p$-values lower than 0.05 (Haaland et al., 1989).

\section{Determination of the mass of CLEAs}

The CLEAs were dried to constant weight using an incubator $\left(45^{\circ} \mathrm{C}\right.$ for $\left.4 \mathrm{~h}\right)$ and were then weighed.

\section{Characterization of synthesized CLEAs microscopy}

The synthesized CLEAs were analyzed and their size was determined using a camera-enabled light microscope (Accu Scope 3310 series coupled to a Micrometrics Premium SE Software version 4). CLEAs were fixed on microscope slides and viewed using $40 \times$ and $100 \times$ objective lenses in a phase contrast mode (Ortiz-Soto et al., 
2009). The aggregates formed before, during, and after quenching were observed and captured. In addition, after several rounds of reuse of CLEA (repeated catalysis of sucrose to determine the number of reaction cycles that the CLEA can catalyze), the nature and size (in $\mu \mathrm{m}$ ) of the aggregates were also determined.

\section{SEM-EDX}

The microstructure and chemical analysis of the CLEAs were performed using an SEM model TESCAN equipped with Oxford instrument X-Max (EDS), iThemba LABS, Materials Research Department, Cape Town, South Africa. The CLEAs were dried to a constant weight $\left(45^{\circ} \mathrm{C}\right.$ for $\left.4 \mathrm{~h}\right)$ prior to the SEM-EDX analysis.

Analysis of secondary structural features of enzyme in CLEAs and free state by FTIR spectroscopy

FTIR spectroscopy was used in the analysis of changes in the secondary structure of the enzyme in the CLEAs and free state. Sample preparation involved dispersion of CLEAs (dried pellet) or free enzyme (lyophilized) in potassium bromide $(1: 10 \mathrm{w} / \mathrm{w})$ followed by homogenization (in a mortar and pestle) before inserting them into disks using the hydraulic gauges. The spectral data were collected in the range of $4000-400 \mathrm{~cm}^{-1}$ using an FTIR spectrophotometer (Thermo Scientific Nicolette iD1, Thermo Electron Corporation, USA). The secondary derivative of peak frequencies in the amide I region (1600-1700 $\mathrm{cm}^{-1}$ ) (performed using SpectraGryph version 1.2) (Susi and Byler, 1986; Wang et al., 2017) was resolved, identified, and fitted with Gaussian curves. From the best fit, the peak areas (from the full width at half maximum) were observed using the OriginPro 2017 software.

\section{Purification of synthesized sugars}

The granular form of activated charcoal (80 g) (Sigma-Aldrich, USA, G-60, 100-350 mesh) was used as the adsorbent of choice used in the purification experiments (extra pure), with a mean particle diameter of $1.5 \mathrm{~mm}$. Before filling the column, activated charcoal was washed in distilled water and autoclaved to remove any residual particles and air from the pores (Nobre et al., 2012). Next, the charcoal was loaded into a glass column $(2.0 \times$ $20 \mathrm{~cm}$ ) that was previously equilibrated with distilled water. The supernatant (from the CLEA catalysis and the whole cell biocatalysis) was applied to the column and eluted with distilled water to remove the nonad- sorbed sugars. The adsorbed sugars (desorption) were eluted using ethanol from $10 \%(\mathrm{v} / \mathrm{v})$ up to $50 \%(\mathrm{v} / \mathrm{v})$ in a stepwise manner. The concentration of reducing sugars in fractions was determined using the dinitrosalicylic acid (DNS) reagent (Miller, 1959). The fractions collected in the desorption phase were evaporated at $60^{\circ} \mathrm{C}$ to remove ethanol and to concentrate the sugars present. Further concentration was performed by freezedrying and the samples were stored at $4{ }^{\circ} \mathrm{C}$ until further use (Nobre et al., 2012).

\section{Analysis of sugars produced \\ from sucrose biocatalysis using the CLEAs \\ Fourier Transform Infrared Spectroscopy of sugars and chemometrics}

All samples (synthesized sugars and standard FOS) were stirred using a stirrer (Sybron/Thermolyne, Iowa, USA) for $1 \mathrm{~h}$ at room temperature $\left(25^{\circ} \mathrm{C}\right)$ and then incubated for $24 \mathrm{~h}$ at $40^{\circ} \mathrm{C}$ and analyzed using FTIR spectrometer (Thermo Scientific Nicolette iD1). Each FTIR spectrum of both the standard and test samples was recorded in the range of $500-4000 \mathrm{~cm}^{-1}$. To identify the carbohydrate-specific fingerprint region and calibrate the chemometric method for quantification of the sugars, different concentrations (six levels) of standard FOS, glucose, sucrose, and fructose solutions (Table 2) were prepared and then analyzed by using FTIR spectrometer. The FTIR spectra of the reaction products of fructosyltransferase CLEA on sucrose were also recorded in the same conditions (Duarte et al., 2002) (Table 2). The calibration set contained 13 quaternary mixtures, 9 ternary mixtures, 3 double mixtures, and 4 single solutions based on an experimental design using Design Expert software (Table 2). The spectra were processed using an IR solution Software Overview (Shimadzu) and Origin ${ }^{R}$ 7SR1 Software (OriginLab Corporation, Northampton, USA). The synthesized FOSs were characterized and quantified using chemometrics via spectral calibration using partial least squares regression with the aid of Unscrambler $\mathrm{X}$ version 10 software (CAMO Software Inc, Oslo, Norway) (Trollope et al., 2015).

\section{Studies on the synthesized FOSS}

Preliminary screening for FOS fermenting potential of the Lactobacillus sp.

A modified method described by Kaplan and Hutckins (2000) was used in the investigation of the utilization of 
Table 2. Concentration in $\%(\mathrm{w} / \mathrm{v})$ of the 4 sugars (glucose, fructose, sucrose and FOS) in the standard solutions of the calibration set

\begin{tabular}{|c|c|c|c|c|}
\hline \multirow{2}{*}{$\mathrm{S} / \mathrm{N}$} & \multicolumn{4}{|c|}{ Sugar concentration \% (w/v) } \\
\hline & glucose & fructose & sucrose & FOS \\
\hline 1 & 5.0 & 5.0 & 50.0 & 5.0 \\
\hline 2 & 10.0 & 5.0 & 25.0 & 10.0 \\
\hline 3 & 5.0 & 10.0 & 25.0 & 10.0 \\
\hline 4 & 25.0 & 5.0 & 10.0 & 5.0 \\
\hline 5 & 10.0 & 10.0 & 10.0 & 10.0 \\
\hline 6 & 5.0 & 25.0 & 10.0 & 10.0 \\
\hline 7 & 50.0 & 5.0 & 5.0 & 5.0 \\
\hline 8 & 25.0 & 10.0 & 5.0 & 5.0 \\
\hline 9 & 10.0 & 25.0 & 5.0 & 5.0 \\
\hline 10 & 5.0 & 50.0 & 5.0 & 5.0 \\
\hline 11 & 5.0 & 5.0 & 10.0 & 50.0 \\
\hline 12 & 10.0 & 25.0 & 5.0 & 50.0 \\
\hline 13 & 5.0 & 5.0 & 5.0 & 50 \\
\hline 14 & & 5.0 & 75.0 & 5 \\
\hline 15 & & 10.0 & 50.0 & \\
\hline 16 & & 50.0 & 10.0 & 10.0 \\
\hline 17 & 5.0 & 75.0 & & \\
\hline 18 & 10.0 & 50.0 & & 10.0 \\
\hline 19 & 50.0 & 10.0 & & 10.0 \\
\hline 20 & 50.0 & 10.0 & & 10.0 \\
\hline 21 & 75.0 & & 10.0 & 10.0 \\
\hline 22 & 10.0 & & 50.0 & 10.0 \\
\hline 23 & & 10.0 & 5.0 & 75.0 \\
\hline 24 & & & 10.0 & 50.0 \\
\hline 25 & 5.0 & 10.0 & & 75.0 \\
\hline 26 & 100.0 & & & \\
\hline 27 & & & & 100.0 \\
\hline 28 & & 100.0 & & \\
\hline 29 & & & 100.0 & \\
\hline
\end{tabular}

The sugar concentrations were varied between $5-100 \%(\mathrm{w} / \mathrm{v})$

FOS by the probiotic bacteria. Briefly, the potential of the probiotic strain in the fermentation of FOSs was investigated using a bromocresol purple amended Man Ragosa Sharp (MRS) agar medium. An MRS-FOS agar medium was prepared by adding $2 \%$ (w/v) purified FOS to MRS agar containing $0.05 \% \mathrm{~L}$-cysteine, $1.5 \%$ agar, and 30 mg of bromocresol purple per liter of medium. The MRS basal medium (i.e., MRS without carbohydrate) was autoclaved and the FOS was filter-sterilized and added to the tempered agar. The probiotic strain was initially grown in MRS broth and then diluted and spread onto the MRS-FOS agar plates. 
Table 3. Summary of CLEA of fructosyltransferase aggregation yields for different solvents used for the catalysis over 4 cycles

\begin{tabular}{|c|c|c|c|c|}
\hline \multirow{2}{*}{ Precipitant } & \multicolumn{4}{|c|}{ Aggregation yield } \\
\hline & $40 \%$ & $50 \%$ & $60 \%$ & $70 \%$ \\
\hline \multicolumn{5}{|c|}{ Ammonium sulphate } \\
\hline $1^{\text {st }}$ cycle & $156 \pm 12$ & $127 \pm 8$ & $142 \pm 18$ & $175 \pm 12$ \\
\hline $2^{\text {nd }}$ cycle & $158 \pm 13$ & $113 \pm 12$ & $134 \pm 9$ & $157 \pm 23$ \\
\hline $3^{\text {rd }}$ cycle & $97 \pm 14$ & $79 \pm 12$ & $81 \pm 15$ & $99 \pm 20$ \\
\hline $4^{\text {th }}$ cycle & $57 \pm 10$ & $60 \pm 9$ & $64 \pm 11$ & $100 \pm 11$ \\
\hline \multicolumn{5}{|l|}{ Toluene } \\
\hline $1^{\text {st }}$ cycle & $173 \pm 25$ & $190 \pm 29$ & $169 \pm 21$ & $179 \pm 23$ \\
\hline $2^{\text {nd }}$ cycle & $195 \pm 13$ & $172 \pm 15$ & $128 \pm 16$ & $109 \pm 17$ \\
\hline $3^{\text {rd }}$ cycle & $93 \pm 17$ & $106 \pm 21$ & $45 \pm 25$ & $100 \pm 27$ \\
\hline $4^{\text {th }}$ cycle & $44 \pm 7$ & $68 \pm 13$ & $12 \pm 2$ & $11 \pm 0$ \\
\hline \multicolumn{5}{|l|}{ Acetone } \\
\hline $1^{\text {st }}$ cycle & $130 \pm 11$ & $61 \pm 9$ & $68 \pm 5$ & $74 \pm 12$ \\
\hline $2^{\text {nd }}$ cycle & $31 \pm 9$ & $24 \pm 6$ & $30 \pm 8$ & $35 \pm 10$ \\
\hline $3^{\text {rd }}$ cycle & $5.4 \pm 0.4$ & $8.0 \pm 2.3$ & $22 \pm 8$ & $22 \pm 2$ \\
\hline $4^{\text {th }}$ cycle & $3.0 \pm 1.0$ & $4.0 \pm 1.0$ & $16 \pm 2$ & $16 \pm 3$ \\
\hline
\end{tabular}

Table 4. Full experimental Box-Behnken design with coded and actual level of variables and the response function for CLEA aggregation yield

\begin{tabular}{c|c|c|c|c|c|c|c|c}
\hline \multirow{2}{*}{ Variables } & \multicolumn{2}{|c|}{$\mathrm{pH}$} & \multicolumn{2}{c|}{ Temperature } & \multicolumn{2}{c}{ Glutaraldehyde } & \multicolumn{2}{c}{ Aggregation yield } \\
\hline std. ord. & coded & real & coded & real & coded & real & experimental & predicted \\
\hline 1 & -1 & 3.0 & -1 & 4 & 0 & 5.5 & 18.0 & 36.4 \\
\hline 2 & -1 & 6.0 & +1 & 4 & 0 & 5.5 & 86.0 & 79.9 \\
\hline 3 & +1 & 3.0 & -1 & 60 & 0 & 5.5 & 39.0 & 45.1 \\
\hline 4 & +1 & 6.0 & +1 & 60 & 0 & 5.5 & 129.0 & 110.6 \\
\hline 5 & -1 & 3.0 & 0 & 32 & -1 & 1.0 & 0 & 14.4 \\
\hline 6 & -1 & 6.0 & 0 & 32 & +1 & 1.0 & 0 & 38.9 \\
\hline 7 & +1 & 3.0 & 0 & 32 & -1 & 10 & 95.0 & 56.1 \\
\hline 8 & +1 & 6.0 & 0 & 32 & +1 & 10 & 155.0 & 140.6 \\
\hline 9 & 0 & 4.5 & -1 & 4 & -1 & 1.0 & 0 & -32.8 \\
\hline 10 & 0 & 4.5 & -1 & 60 & +1 & 1.0 & 0 & -20.5 \\
\hline 11 & 0 & 4.5 & +1 & 4 & -1 & 10 & 11.0 & 31.5 \\
\hline 12 & 0 & 4.5 & +1 & 60 & +1 & 10 & 26.0 & 58.8 \\
\hline 13 & 0 & 4.5 & 0 & 32 & 0 & 5.5 & 191.0 & 197.8 \\
\hline 14 & 0 & 4.5 & 0 & 32 & 0 & 5.5 & 203.0 & 197.8 \\
\hline 15 & 0 & 4.5 & 0 & 32 & 0 & 5.5 & 198.0 & 197.8 \\
\hline 16 & 0 & 4.5 & 0 & 32 & 0 & 5.5 & 200.0 & 197.8 \\
\hline 17 & 0 & 4.5 & 0 & 32 & 0 & 5.5 & 197.0 & 197.8 \\
\hline & & & & & & & &
\end{tabular}


Table 5. Analysis of variance (ANOVA) for the Box-Behnken quadratic model for aggregation yield by the CLEA

\begin{tabular}{l|c|c|c|c|c}
\hline \multicolumn{1}{c|}{ Source } & Sum of squares & DF & $F$-value & Prob $>F$ & \\
\hline Model & $1.072 \times 10^{5}$ & 9 & 11.50 & 0.0020 & significant \\
\hline $\mathrm{A}$ & 5940.50 & 1 & 5.74 & 0.0478 & significant \\
\hline $\mathrm{B}$ & 780.13 & 1 & 0.75 & 0.4142 & not significant \\
\hline $\mathrm{C}$ & 10296.13 & 1 & 9.94 & 0.0161 & significant \\
\hline $\mathrm{A}^{2}$ & 6168.32 & 1 & 5.96 & 0.0447 & significant \\
\hline $\mathrm{B}^{2}$ & 35270.84 & 1 & 34.05 & 0.0006 & significant \\
\hline $\mathrm{C}^{2}$ & 39637.27 & 1 & 38.27 & 0.0005 & significant \\
\hline $\mathrm{AB}$ & 121.00 & 1 & 0.12 & 0.7425 & not significant \\
\hline $\mathrm{AC}$ & 900 & 1 & 0.87 & 0.3823 & not significant \\
\hline BC & 56.25 & 1 & 0.054 & 0.8224 & not significant \\
\hline Residual & 7250.55 & 7 & & & \\
\hline Lack of fit & 78.80 & 3 & 121.35 & 0.02 & not significant \\
\hline Pure error & 7171.75 & 4 & & & \\
\hline Correlation total & $1.145 \times 10^{5}$ & 6 & & & \\
\hline
\end{tabular}

Coefficient of variation $=35.34 ; R^{2}=0.94 ;$ Adjusted $R^{2}=0.85$; Adequate precision $=9.34 ; \mathrm{A}-\mathrm{pH}$; $\mathrm{B}$ - temperature; $\mathrm{C}$ - glutaraldehyde; $\mathrm{DF}$ - degree of freedom

\section{Prebiotic effect of Aureobasidium sp. FOS in vitro}

The method described by Li et al. (2008) was used in the investigation of the prebiotic properties of the newly synthesized FOSs with minor modifications. Lactobacillus sp. was incubated for $48 \mathrm{~h}$ at $37^{\circ} \mathrm{C}$ and the stock cultures were stored in $10 \%$ glycerol at $4{ }^{\circ} \mathrm{C}$ until needed. MRS broth amended with $0.5 \mathrm{~g} / 1 \mathrm{~L}$-cysteine hydrochloride, $0.2 \mathrm{~g} / 1$ of sodium thioglycolate, and $0.1 \mathrm{~g} / 1$ of $\mathrm{CaCl}_{2}$ $2 \mathrm{H}_{2} \mathrm{O}$ was used as the stock culture medium and as the basal medium. Basal MRS broth supplemented with $0.5 \%, 1 \%$, and $2 \%$ (w/v) of $A$. pullulan FOS and inoculated with $24 \mathrm{~h}$ culture of the probiotic bacteria and incubated at $37^{\circ} \mathrm{C}$. The effects of $A$. pullulan FOS on bacterial growth was studied by cultivating the Lactobacillus sp. for $24 \mathrm{~h}$ in the basal medium and further inoculation into the basal medium containing the FOS. The bacteria were then cultured under anaerobic conditions (using anaerobic GasPack, Oxoid, Thermo Fisher) for $24 \mathrm{~h}$ at $37^{\circ} \mathrm{C}$. Thereafter, the culture broth was centrifuged at $3000 \times g$ for $20 \mathrm{~min}$. The pellets obtained were washed several times with phosphate buffered saline (PBS) (0.1 M phosphate buffer pH 7.4, 0.9\% saline) and then were diluted appropriately. The growth of the bacterial cells via turbidity was measured at $600 \mathrm{~nm}$. All measurements were performed in triplicate (Li et al., 2008).

\section{Statistical analysis}

The data obtained were analyzed using one-way ANOVA and significant differences were determined by Duncan's multiple range test. Differences were considered significant at $P<0.05$. Statistical analyses were performed using the GraphPad Prism program version 7.0.

\section{Results}

\section{Screening of the best precipitant} and its concentration for CLEA production

Of the several precipitants (toluene, acetone, and ammonium sulfate) tested, ammonium sulfate was found to be the best as it retained nearly $70 \%$ residual activity after four catalytic cycles. Toluene exhibited about $65 \%$ residual activity, whereas acetone and ethanol exhibited less than $40 \%$ residual activity after four catalytic cycles (Table 3 ).

\section{Statistical optimization of medium for CLEA production}

Following were the optimum conditions obtained after the verification of the RSM model: $5 \%$ (v/v) glutaraldehyde, $\mathrm{pH} 5.5$, and $32^{\circ} \mathrm{C}$. They corresponded to $190 \%$ residual activity after the first reaction cycle. Table 4 shows the full experimental design (BBD) with coded and actual levels of variables and the response function. 


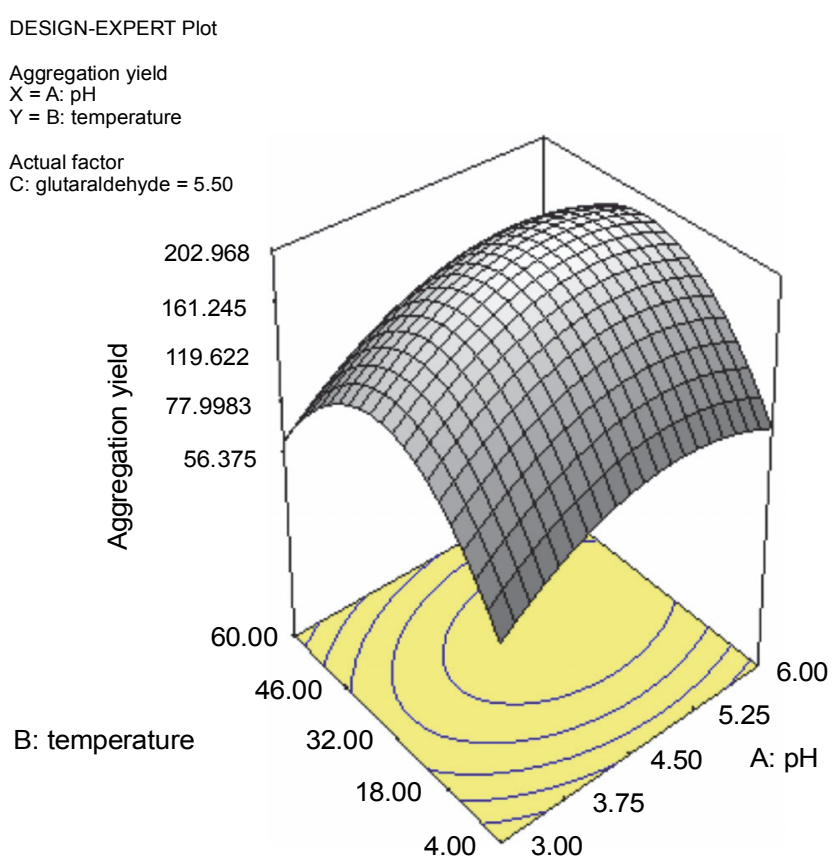

Fig. 1. 3-D response surface plot showing the interaction between the variables, $\mathrm{pH}$ and temperature on the aggregation yield

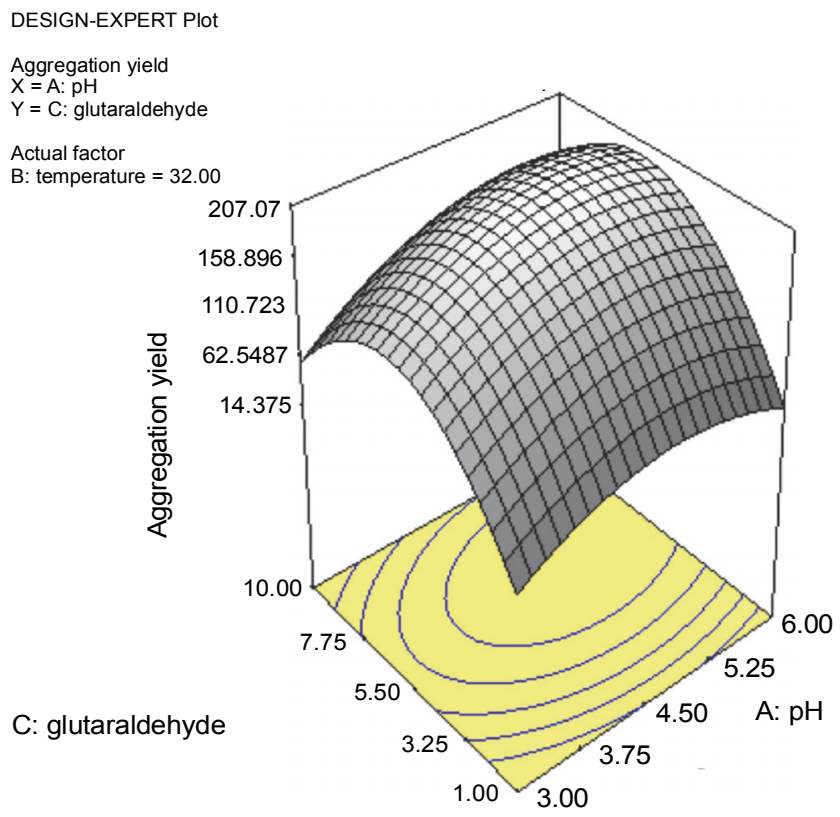

Fig. 2. 3-D response surface plot showing the interaction between the variables, glutaraldehyde and $\mathrm{pH}$ on the aggregation yield

Table 5 shows the ANOVA for the quadratic model for the residual activity of CLEA catalysis. The $R^{2}, F$ value, adjusted precision, and CV were found to be $0.94,11.50$, 9.34 , and 35.34 respectively. Figures $1-3$ show the response surface plots showing the interaction of these parameters.

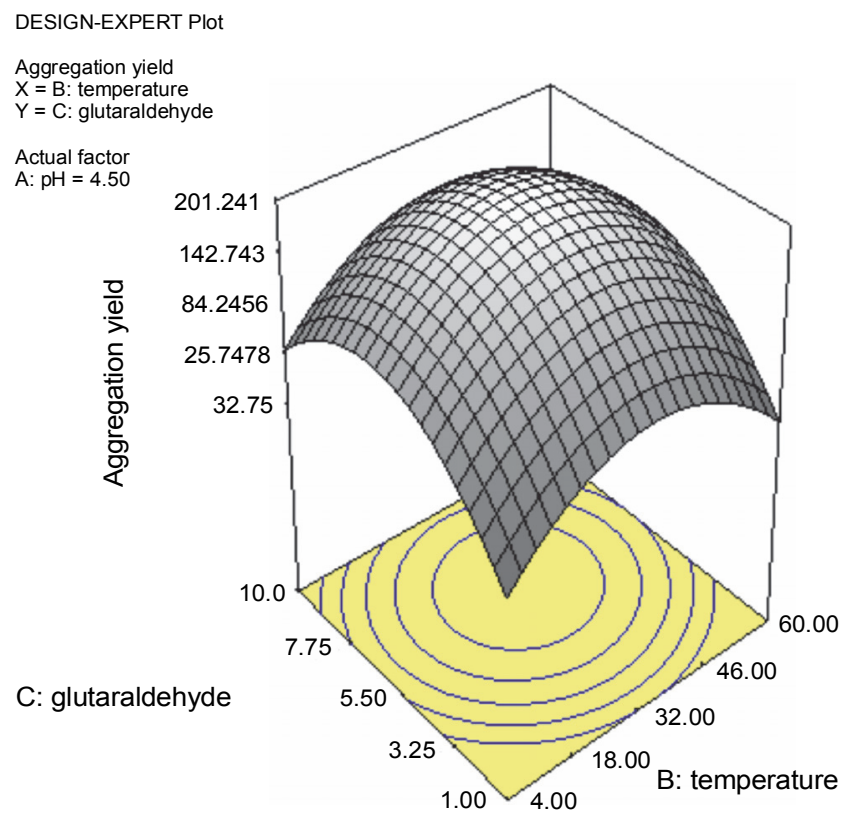

Fig. 3. 3-D response surface plot showing the interaction between the variables, glutaraldehyde and temperature on the aggregation yield

\section{Characterization of CLEA}

The aggregation before and after catalysis has been visualized using photomicrography (Fig. 4). The size of aggregates before catalysis varied from 10 to $20 \mu \mathrm{m}$. The SEM-EDX analysis revealed that the size of aggregates (CLEAs) after catalysis was uneven in size, which varied from 10 to $40 \mu \mathrm{m}$ (Fig. 5). The elemental composition of the CLEAs revealed the presence of phosphorus (30\%), sulfur $(37.09 \%)$, copper $(0.17 \%)$, zinc $(0.94 \%)$, oxygen (28.66\%), calcium (1.4\%), and silver (0.68\%) (Fig. 6).

\section{Protein's secondary structure in the CLEAs and free state}

Figure 7 shows the FTIR spectral data for the CLEAs and free enzyme. Figures 8 and 9 show the curve fitting process for the free and CLEAs. The content of alpha helix, beta sheet, beta turn, and random coils of the free enzyme were $21.3,5.4,54.4$, and $9.1 \%$, respectively, whereas the content of alpha helix, beta sheet, beta turn, and random coils of the free CLEAs were 3.7, 65.4, 25.3, and $6.7 \%$, respectively (Table 6).

\section{Chemometric analysis of FTIR spectral data for the biocatalysis of sucrose}

The production of FOSs by the CLEA biotransformation of sucrose was confirmed by FTIR analysis. The FTIR spectra of standards such as glucose, fructose, su- 


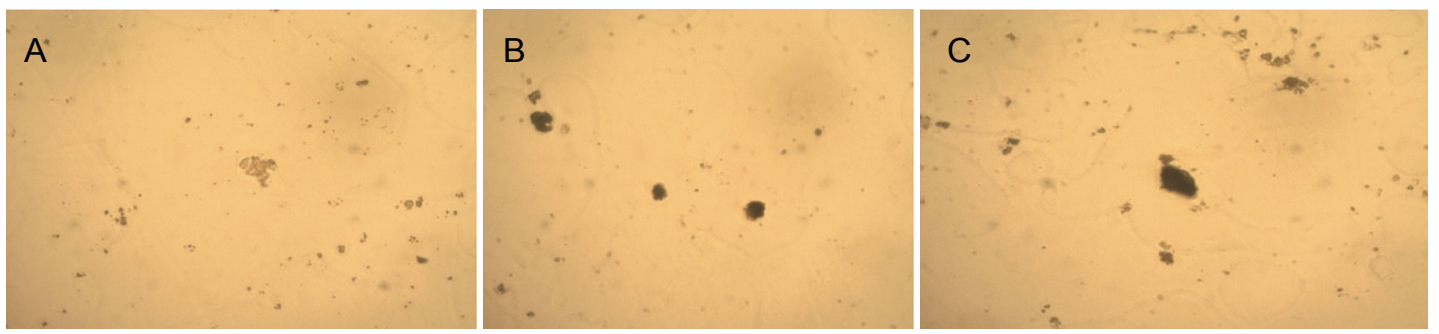

Fig. 4. Photomicrography of the CLEA: A) before quenching B) after quenching and C) after several rounds of catalysis

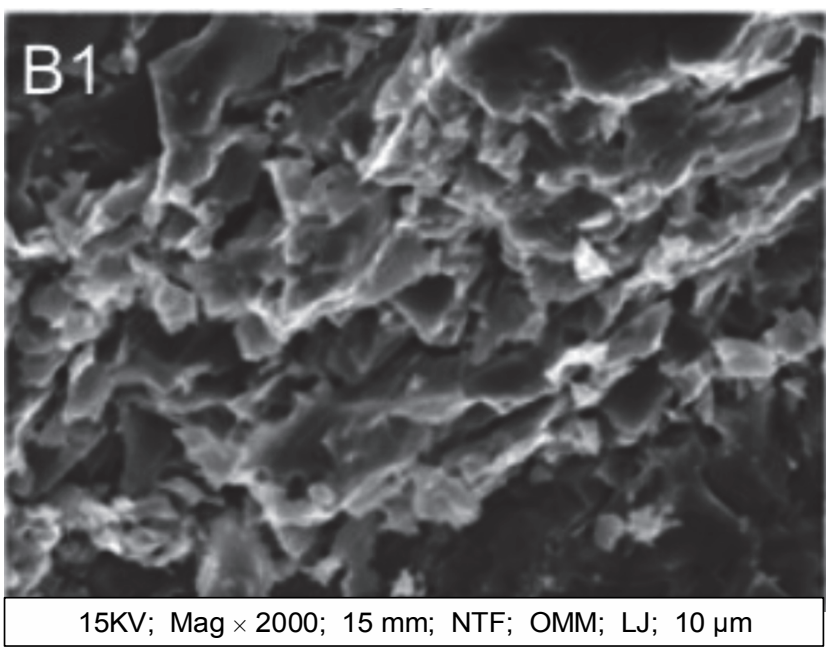

Fig. 5. Scanning electron microscopy image of the CLEA

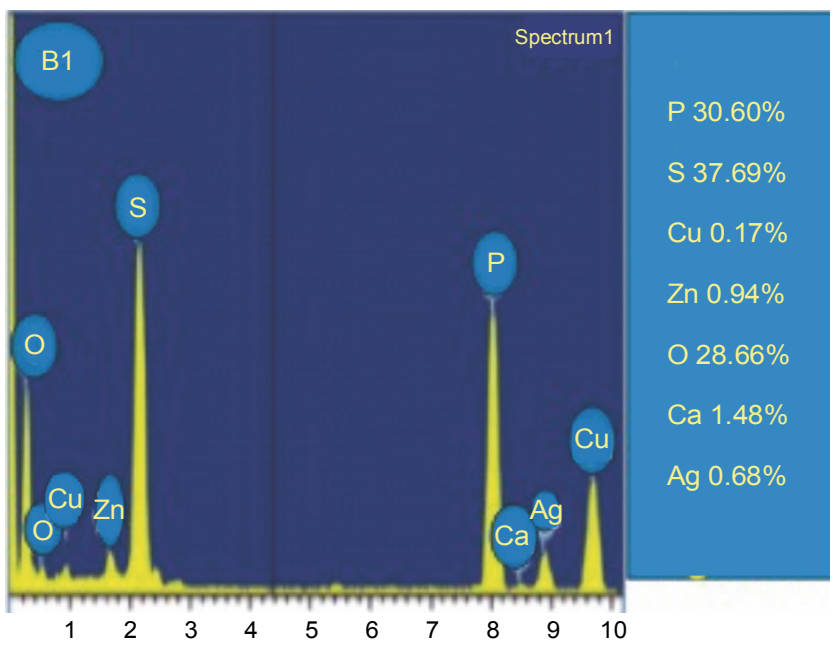

Fig. 6. Energy dispersive X-ray analysis to estimate the elemental composition of the CLEA

crose, and FOS were compared with the purified reaction products after purification performed using an activated charcoal chromatography. Figure 10 shows the comparative FTIR spectra of the pure component stan- dard sugars and the purified reaction products of sucrose biocatalysis by the CLEAs. Figure 11 shows the principal component analysis. Table 7 shows the summary of the latent variables obtained, as well as the root mean square values for both the raw spectra and the first derivative (Savistsky-Golay). Table 8 shows the quantification of total FOSs, sucrose, glucose, and fructose by the CLEA.

\section{Prebiotic potential of FOSS}

\section{Preliminary screening for FOS utilization} on bromo cresol purple amended MRS agar

FOSs changed the color of MRS agar plate from purple to light yellow after fermentation by Lactobacillus sp. (Fig. 12).

\section{Effect of newly synthesized FOS, commercial FOS, and glucose on growth and $\mathrm{pH}$ \\ of probiotic bacterial strain}

The bacterial population growth increased when newly synthesized FOS were used, as evidenced by the value of turbidity (optical density (OD at $660 \mathrm{~nm}$ ) of 0.58 . The negative control (bacteria grown on MRS only) and control (bacteria grown on MRS + glucose) had a relatively low-bacterial population $\left(\mathrm{OD}_{660 \mathrm{~nm}}\right.$ of 0 and 0.1 , respectively). The commercial FOS showed a comparable bacterial growth with the newly synthesized FOS at $1 \%(\mathrm{w} / \mathrm{v})$ with an $\mathrm{OD}_{660 \mathrm{~nm}}$ of 0.6 (Fig. 13).

\section{Effect of the incubation period}

on the prebiotic potential of FOS on Lactobacillus sp.

A steady decrease in the $\mathrm{pH}$ of the medium containing FOS (from 5.6 to 4.3 ) after $24 \mathrm{~h}$ incubation was observed, followed by a steady $\mathrm{pH}$ (4.5) up to $48 \mathrm{~h}$. There was a steady increase in the turbidity $\left(\mathrm{OD}_{660 \mathrm{~nm}}\right.$ value of 0.2 to 0.58 ) after $28 \mathrm{~h}$ incubation (plateau phase) followed by a steady increase in the growth rate $\left(\mathrm{OD}_{660 \mathrm{~nm}}\right.$ value of 0.58$)$ for the next $24 \mathrm{~h}$ (Fig. 14). In 


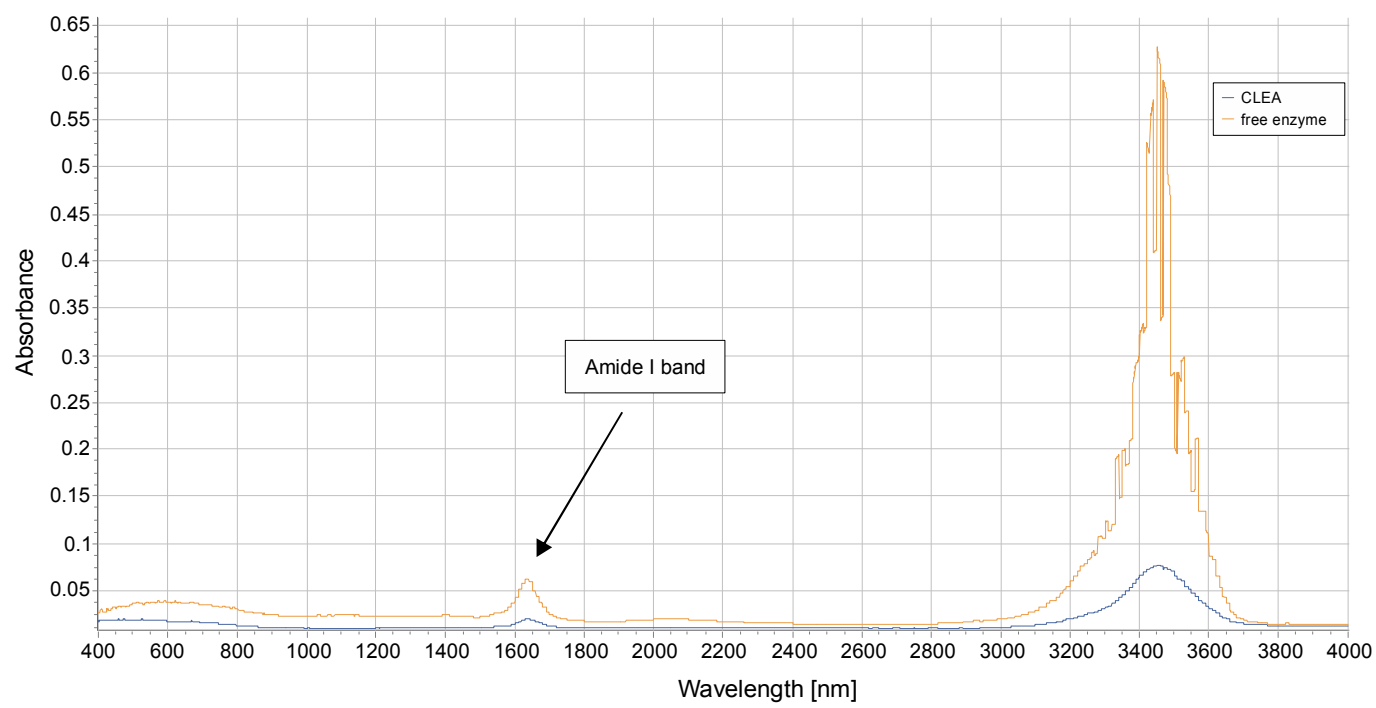

Fig. 7. Fourier Transform InfraRed Spectra for the cross-linked enzyme aggregate and the free enzyme

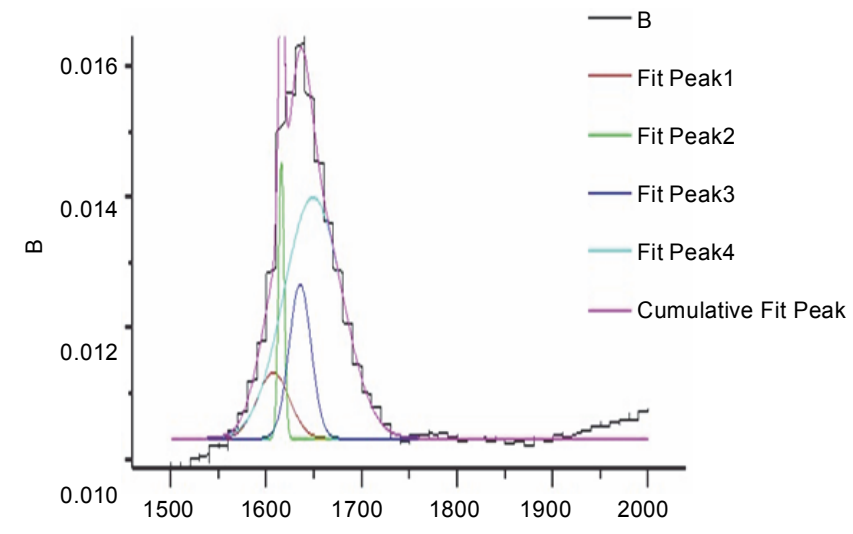

Fig. 8. Gaussian curve fitting showing the several peaks in the deconvoluted amide I region $\left(1600-1700 \mathrm{~cm}^{-1}\right)$ for free enzyme; Fit Peak 1: beta sheet, Fit Peak 2: alpha helix, Fit Peak 3: random coil, Fit Peak 4: beta turn

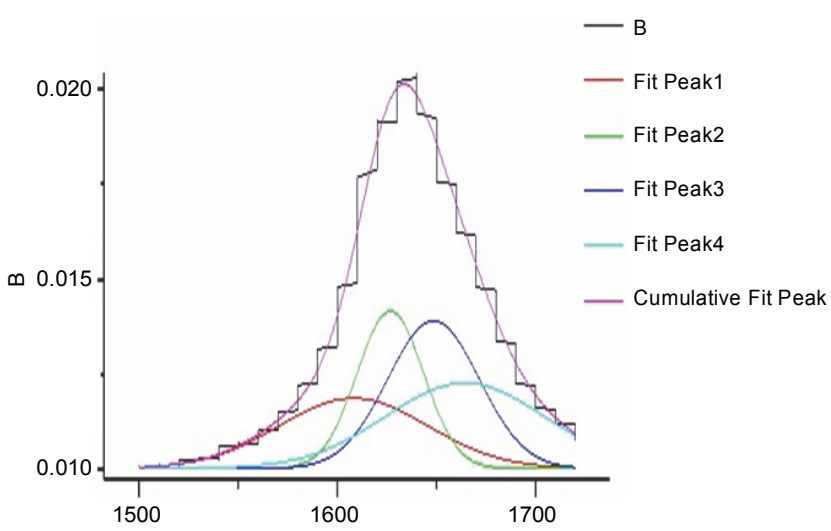

Fig. 9. Gaussian curve fitting showing the several peaks in the deconvoluted amide I region (1600-1700 $\mathrm{cm}^{-1}$ ) for crosslinked enzyme aggregate; Fit Peak 1: beta sheet, Fit Peak 2: alpha helix, Fit Peak 3: random coil, Fit Peak 4: beta turn case of the medium without FOS, the plateau phase was reached at $36 \mathrm{~h}$ (Fig. 15).

\section{Discussion}

In this study, the CLEAs of fructosyltransferase were prepared and statistically optimized for the biocatalysis of sucrose to yield FOSs. The FOSs were quantified using chemometrics and their potential use as prebiotics was investigated. The statistical approach to optimization is of immense industrial importance, especially for scale-up processes. To the best of our knowledge, there is little or no information regarding the use of a carrierfree immobilization technique for fructosyltransferases. Hence, our study is the first of its kind in that regard.

During the screening of precipitants, we focused on common protein precipitants, namely, ammonium sulfate, acetone, and ethanol at a concentration ranging from 40 to $70 \%$ (v/v). During the process of aggregation, ammonium sulfate was found to be the best precipitant; however, toluene was also found to be a good precipitant in comparison with ammonium sulfate. During the conduct of this study, neither toluene nor ethanol could fully precipitate the enzyme prior to the process of crosslinking with glutaraldehyde; therefore, they showed lower recovery than that of ammonium sulfate. Furthermore, under repeated catalysis, the CLEAs produced using ammonium sulfate had a higher recovery after four rounds of reuse than that of toluene and ethanol. This suggests that ammonium sulfate is good as the cross- 
Table 6. The content of various structural motifs in predicted protein secondary structures of amide I band in the free enzyme and the cross-linked enzyme aggregates

\begin{tabular}{l|c|c|c|c}
\hline \multirow{2}{*}{} & \multicolumn{4}{|c}{ Secondary structures } \\
\cline { 2 - 5 } & $\alpha$-helix [\%] & $\beta$-sheet [\%] & $\beta$-turn [\%] & random coil [\%] \\
\hline Free enzyme & 21.3 & 15.4 & 54.4 & 9.1 \\
\hline CLEA & 3.7 & 65.4 & 25.3 & 6.7 \\
\hline
\end{tabular}

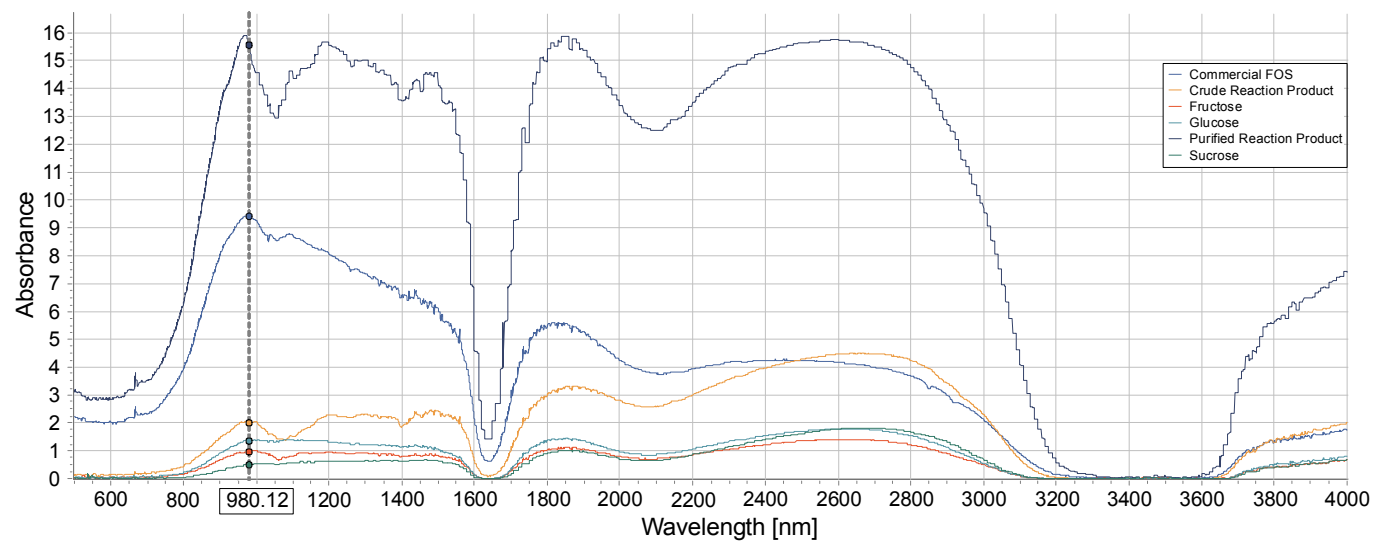

Fig. 10. Comparative FTIR spectra of standard sugars (sucrose, glucose, fructose and fructooligosaccharides) and the reaction products of sucrose transfructosylation (crude and activated charcoal-purified)

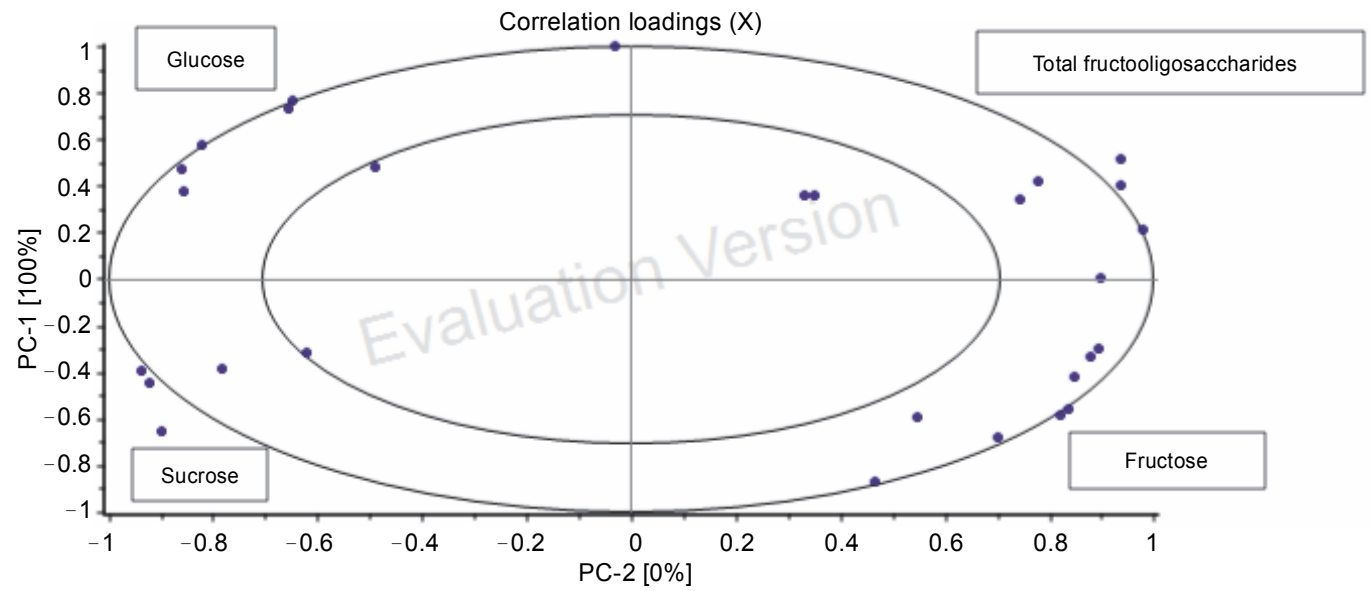

Fig. 11. Scores scatter plot of the two first principal components, PC1 and PC2 obtained for the set of standard sugar solutions

linker compared to the other organic solvents. There was a gradual loss in the activity of the enzyme as the number of rounds of the catalysis increased. This might have been due to the bioaccumulation of the products of sucrose catalysis. The ammonium sulfate-CLEA was able to maintain its $100 \%$ residual activity over four rounds of catalysis. This is quite remarkable as other precipitants such as toluene and ethanol showed a loss of more than $50 \%$ and $90 \%$ activity, respectively. In addition, in the initial choice of the concentration of cross-linker, we found that a high concentration of glutaraldehyde (up to $10 \%$ ) had very a negligible adverse effect on CLEA catalysis. The amount of glutaraldehyde used for CLEA production reported in the recent literature was $0.125 \%$ v/v (Ortiz-Soto et al., 2009). According to our results, we noted that at this concentration, there was no formation of CLEA and biocatalysis was found to be very poor (result not shown). High amounts of glutaraldehyde have 
Table 7. The application of partial least regression square to the four sets of data for calibration of the sugars

\begin{tabular}{c|c|c|c|c}
\hline & Fructooligosaccharide & Fructose & Glucose & Sucrose \\
\hline Raw Spectra & 8 & 7 & 8 & 7 \\
\cline { 2 - 5 } RMSEP [\%] & 8.4 & 11.2 & 10.8 & 11.4 \\
\hline $\begin{array}{c}\text { First Derivative } \\
\text { RMSEP [\%] }\end{array}$ & 5 & 4 & 5 & 6 \\
\cline { 2 - 5 } & 3.2 & 2.9 & 3.2 & 6.2 \\
\hline
\end{tabular}

RMSEP - root mean square error

Table 8. Partial least square regression model for the predicted and actual concentrations ( $\% \mathrm{w} / \mathrm{v})$ of the four sugars present in the reaction products of sucrose biocatalysis

\begin{tabular}{c|c|c|c|c|c|c|c|c}
\hline \multirow{2}{*}{} & \multicolumn{2}{|c|}{ Total fructooligosaccharides } & \multicolumn{2}{c|}{ Fructose } & \multicolumn{2}{c}{ Glucose } & \multicolumn{2}{c}{ Sucrose } \\
\cline { 2 - 8 } & $\begin{array}{c}\text { predicted } \\
\%(\mathrm{w} / \mathrm{v})\end{array}$ & $\begin{array}{c}\text { actual } \\
\%(\mathrm{w} / \mathrm{v})\end{array}$ & $\begin{array}{c}\text { predicted } \\
\%(\mathrm{w} / \mathrm{v})\end{array}$ & $\begin{array}{c}\text { actual } \\
\%(\mathrm{w} / \mathrm{v})\end{array}$ & $\begin{array}{c}\text { predicted } \\
\%(\mathrm{w} / \mathrm{v})\end{array}$ & $\begin{array}{c}\text { actual } \\
\%(\mathrm{w} / \mathrm{v})\end{array}$ & $\begin{array}{c}\text { predicted } \\
\%(\mathrm{w} / \mathrm{v})\end{array}$ & $\begin{array}{c}\text { actual } \\
\%(\mathrm{w} / \mathrm{v})\end{array}$ \\
\hline CLEA & $56 \pm 2.1$ & $57 \pm 0.3$ & $12 \pm 1.8$ & $10 \pm 1.8$ & $23 \pm 2.1$ & $22 \pm 2.1$ & $13 \pm 4.1$ & $11 \pm 4.1$ \\
\hline
\end{tabular}

been reported to inhibit the catalysis by CLEA because of the adverse effect of excessive cross-linking (OrtizSoto et al., 2011). However, we postulate that because glutaraldehyde reacts primarily with the $\epsilon$-amino groups of lysine and other groups such as hydroxyl, secondary amino, and alpha-amino groups (Migneault et al., 2004), the $A$. pullulans fructosyltransferase might contain fewer lysine residues than that of other enzymes used so far to prepare CLEAs; therefore, there is a need for a higher amount of glutaraldehyde to prepare CLEAs.

In this study, a statistical method was employed to optimize the operational conditions for CLEA biocatalysis, as well as to optimize the product release. The effects of $\mathrm{pH}$, the concentration of glutaraldehyde, and the effective temperature were statistically optimized using the RSM model. In the BBD model, we observed that reactions with low concentrations of glutaraldehyde showed a low aggregation yield irrespective of the $\mathrm{pH}$ and the temperature (Table 3). ANOVA revealed that the model was statistically significant, and the lack of fit was not significant (Table 4 ). The $\mathrm{CV}, R^{2}$, adequate precision, predicted- $R^{2}$ were $35.34,0.94,9.34$, and -0.0035 , respectively. The regression equation showing this interaction is given in coded units as follows:

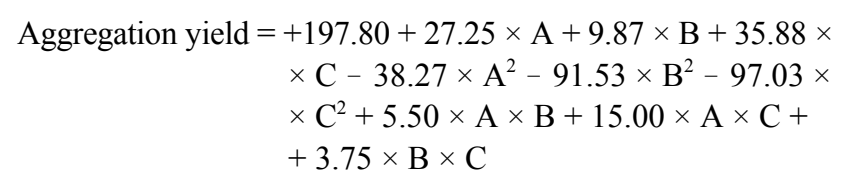

where $A, B$, and $C$ represents $\mathrm{pH}$, temperature, and concentration of glutaraldehyde, respectively.

The three-dimensional (3D) surface plots showed varying degrees of interaction between the variables considered. The responses obtained from the $3 \mathrm{D}$ response surface plots indicate how the numerous interactions that occur between the studied variables affect the aggregation yield, which is very useful in characterizing the responses (Ademakinwa et al., 2017). The optimum conditions statistically derived from the RSM model were found to be $5 \%$ glutaraldehyde, $\mathrm{pH} 5.5$, and $32^{\circ} \mathrm{C}$.

In the response plots of the effects of $\mathrm{pH}$ and temperature, the shape of the curves shows that an increase in the $\mathrm{pH}$ led to an increase in the aggregation yield up to $200 \%$ prior to a decrease. A similar observation was observed for temperature; as the temperature was simultaneously increased, there was an increase in the aggregation yield. At temperature higher than $45^{\circ} \mathrm{C}$, the concomitant effects on the aggregation yield were adverse.

The effects of the concentration of glutaraldehyde and $\mathrm{pH}$ on the aggregation yield resulted in a bell-shaped curve indicating the effects of an increase in either of the variables on the aggregation yield. An initial increase in the concentration of glutaraldehyde $(1-2.5 \% \mathrm{v} / \mathrm{v})$ increased the aggregation yield, a further increase of over $5 \%$ resulted in a loss in the aggregation yield. The same was observed with respect to the effects of $\mathrm{pH}$, in which an increase in the $\mathrm{pH}$ resulted in the initial increase in 


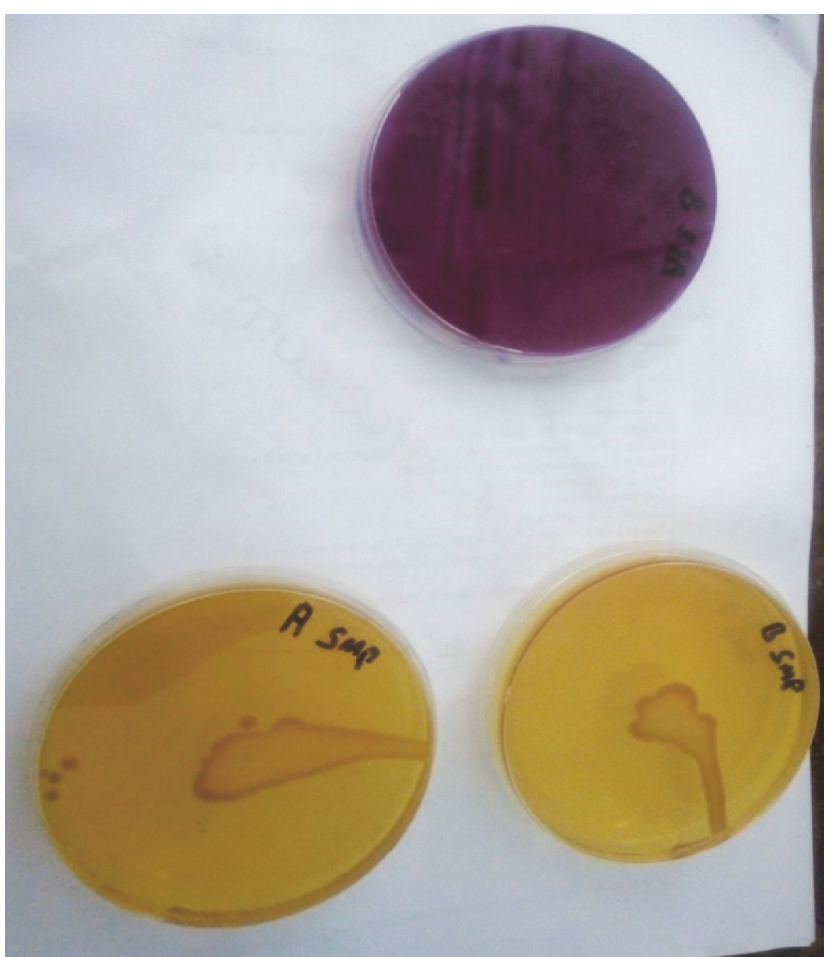

Fig. 12. Utilization of purified $A$. pullulans FOS by Lactobacillus sp. on Bromo cresol-purple amended MRS agar plate

the aggregation yield, but a further increase did not lead to a significant loss in the aggregation yield (Fig. 1-3). The effect of $\mathrm{pH}$ showed a moderate effect on the aggregation yield as the $\mathrm{pH}$ increased. Although few reports exist on the effects of statistically optimized conditions on the potentials of CLEA biocatalysis, some authors have reported that CLEA catalysis can be performed under mild conditions of temperature and $\mathrm{pH}$. Vrsanska et al. (2017) reported that $4{ }^{\circ} \mathrm{C}$ and $\mathrm{pH} 4.0$ were optimum conditions of catalysis for laccase CLEA. It is noteworthy that the effects of $\mathrm{pH}$ on the biocatalysis by CLEA is caused due to the ionic interactions between the enzyme and the cross-linking agent. This often leads to a change in the hydrogen ion concentration, which shifts the optimum $\mathrm{pH}$ between acidic and alkaline conditions (Fernandez-Fernandez et al., 2013).

The effect of concentration of glutaraldehyde (1-10\% $\mathrm{v} / \mathrm{v})$ and temperature $\left(4-60^{\circ} \mathrm{C}\right)$ on the aggregation yield showed varied results when compared with the combined variables discussed above. An initial increase in the central values $\left(\mathrm{pH}-5.5\right.$, temperature $-32^{\circ} \mathrm{C}$ and glutaraldehyde $-5.0 \% \mathrm{v} / \mathrm{v}$ ) led to an increase in the aggregation yield but a further increase above these central values led to a significant decrease in the aggregation yield.

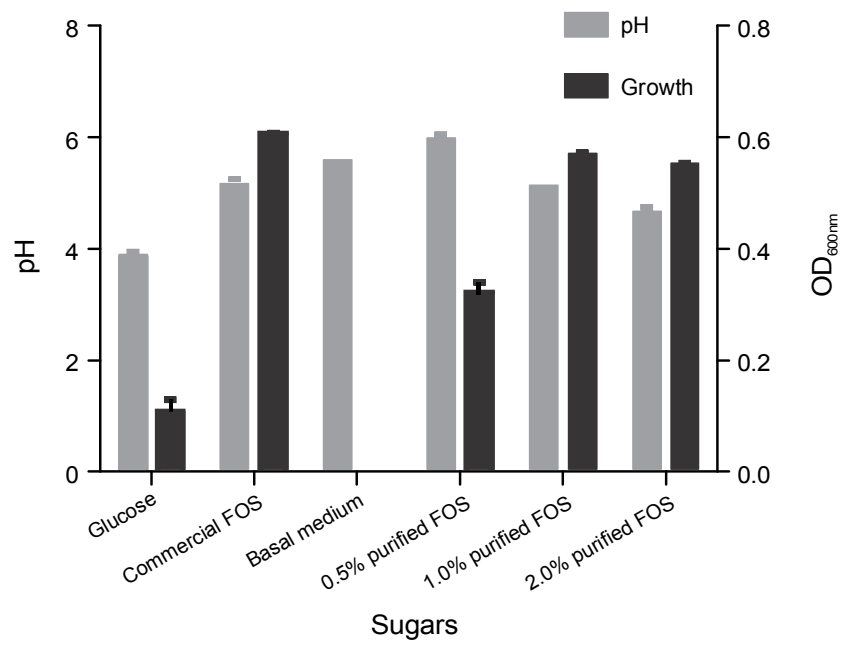

Fig. 13. Effects of different sugars on growth and $\mathrm{pH}$ of Lactobacillus sp.

The combined effect of temperature of up to $60^{\circ} \mathrm{C}$ and increased concentration of glutaraldehyde (up to $5 \% \mathrm{v} / \mathrm{v}$ ) resulted in an increase in the aggregation yield; this might be attributed to the stabilization of linkages that keep the conformation of the catalytic active site of the enzyme (Wilson et al., 2004). Thus, the increased aggregation yield is often a result of a better thermal stability of the CLEAs provided by the coaggregation and crosslinking by the glutaraldehyde. In addition, it is observed that extremes of temperatures might lead to thermal denaturation of CLEAs leading to a loss of their activity (Broun, 1976). The use of the CLEA under the optimized conditions resulted in the repeated reuse of CLEA for catalysis in over six catalytic cycles while still retaining about $70 \%$ of their residual activity. This is albeit less visible with the 10 catalytic cycles obtained when Bacillus subtilis levansucrase (Ortiz-Soto et al., 2009).

The results of SEM-EDX experiments of CLEA showed the morphology, as well as the elemental composition of the CLEA. The sizes of the CLEAs ranged from 10 to $40 \mu \mathrm{m}$, and they were not evenly shaped. The elemental composition of CLEA revealed that they had a higher composition of phosphorus (30.6\%), sulfur (37.7\%), and oxygen (28.7\%) than that of calcium (1.5\%), copper $(0.17 \%)$, zinc $(1.0 \%)$ and silver $(0.7 \%)$. This indicates that CLEA is proteinaceous in nature. This is the first-ever report of the determination of the elemental composition of CLEA using EDX analysis. The size of the CLEA increased after quenching and repeated catalytic cycles. Initially, the microscopic analysis revealed that the size of CLEAs was around $10 \mu \mathrm{m}$, whereas after 


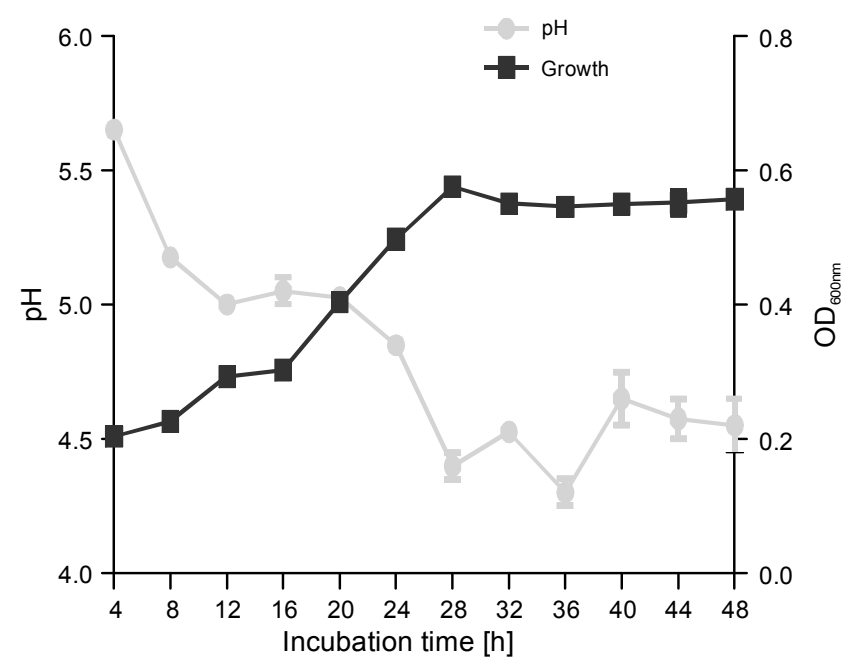

Fig. 14. Effect of fructooligosaccharides on growth and $\mathrm{pH}$ of Lactobacillus sp. at different cultivation time under anaerobic conditions in MRS-FOS containing medium

quenching, the size increased to around $40 \mu \mathrm{m}$. This increase in size might be due to the increased aggregation caused by the process of centrifugation and/or due to the presence of some residual glutaraldehyde which had not been washed off completely (Ortiz-Soto et al., 2009). Ortiz-Soto et al. (2009) reported the effect of quenching on the sizes of the CLEA. Increased sizes (from 20 to $50 \mu \mathrm{m}$ ) were also noted by the levansucrase CLEA after quenching and repeated catalysis (Ortiz-Soto et al., 2009).

To further ascertain the structural features between the free enzyme and CLEAs, the secondary structure of all CLEAs was observed by FTIR spectroscopy, as it is considered an important technique to monitor the conformational changes in proteins (Jung, 2000). The structural variation in the proteins' conformation is mostly studied in the range that constitutes the amide I band, as this is the most intense absorption band for polypeptides (Susi and Byler, 1986). It was observed that there was an increased content of the beta sheet component in the CLEAs compared to the free enzyme. This is the first report in which the secondary structure of fructosyltransferase CLEA is explained using the FTIR spectral data. Wang et al. (2017), predicted the changes in the secondary structure of lipase CLEA; they observed a higher number of beta sheets after aggregation. Thus, we can conclude that cross-linking with glutaraldehyde allowed for the transformation of helical structures and beta sheet to beta turns due to protein aggregation.

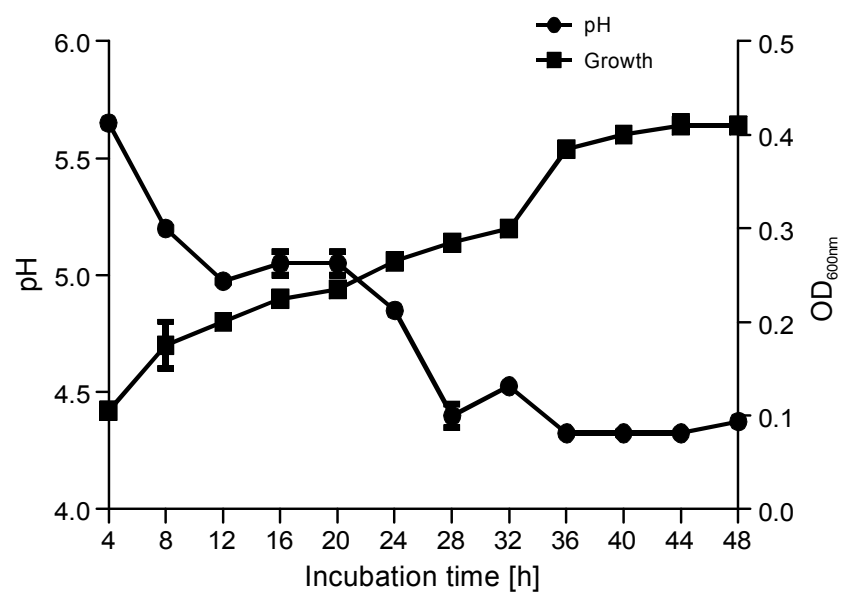

Fig. 15. Growth and pH of Lactobacillus sp. at different cultivation time under anaerobic conditions in Man-Rogosa-Sharp-only medium

The transfructosylation of sucrose catalyzed by fructosyltransferase CLEA resulted in the production of FOS. Figure 8 shows the FTIR spectra of the aqueous solution of each pure sugar component as well as the purified reaction products. The characteristic regions of carbohydrate fingerprint from the FTIR spectra for glucose, sucrose, commercial FOS, fructose, and purified reaction products of FOS had peaked at regions that varied from 1007 to $1193 \mathrm{~cm}^{-1}, 995-1047 \mathrm{~cm}^{-1}$, $969-1039 \mathrm{~cm}^{-1}, 956-1171 \mathrm{~cm}^{-1}$, and $968-1271 \mathrm{~cm}^{-1}$. The crude reaction products had peaked at $969,997.2$, 1078 , and $1095 \mathrm{~cm}^{-1}$. It was observed that the spectral signatures of each sugar were distinguishable. Sugars are known to have exocyclic and endocyclic $\mathrm{C}-\mathrm{O}$ bonds located at $995 \mathrm{~cm}^{-1}$ and $1080 \mathrm{~cm}^{-1}$ for sucrose and glucose/fructose, respectively (Adina et al., 2010). The broad band observed around $3700-3000 \mathrm{~cm}^{-1}$ are fingerprint regions for $\mathrm{H}_{2} \mathrm{O}$ and $-\mathrm{OH}$ absorption frequencies. There are other fingerprint regions for other functional groups such as peaks that correspond to stretching vibrations of - $\mathrm{C}-\mathrm{H}$ inside $\mathrm{CH}_{3}$ or $\mathrm{CH}_{2}$ groups. Characteristically, a unique peak that exists for the FOS was found in the $965 \mathrm{~cm}^{-1}$ region. This peak was found in the FTIR spectra of the commercial FOS, crude, and purified reaction products, and it was noticeably absent in sucrose and glucose. This is a nondestructive and nontoxic confirmation of the transfructosylation of sucrose to FOS by the CLEA.

The physiological and biotechnological importance of FOS necessitated the investigations of the use of FOS 
from $A$. pullulans NAC8 as a prebiotic source. There is an increasing trend in the addition of FOS in various food products because they possess prebiotic properties (Bali et al., 2015, Flores-Maltos et al., 2015). Their consumption increases fecal bolus and the frequency of depositions and reduces constipation, which is a problem existing in the modern world in humans and in infants (early months) (Sabater-Molina et al., 2009).

In this study, bacterial strains that were able to perform the process of fermentation of FOS (and possibly produced acid end products) grew as colonies surrounded by yellow zones against a purple background. The use of bromocresol purple as an indicator of utilization of FOS by probiotic strains served as a necessary yardstick needed for quick preliminary screening of these strains. As reported in this study, the newly synthesized FOS, as well as the commercially obtained FOS, had colonies that existed as a yellow zone against a purple background. This agrees with the report of Kaplan and Hutckins (2000), where several Lactobacillus sp. were found to utilize FOS concomitantly forming yellow colonies.

Investigations into the prebiotic effectiveness (i.e., how the growth of beneficial bacteria is enhanced) of A. pullulans FOS on Lactobacillus sp. revealed that $1 \%$ (w/v) FOS had a slight stimulatory effect on the growth of bi dobacteria compared with the controlled medium. In addition, the $\mathrm{pH}$ values of the media dropped with an increase in the mass of bi dobacteria. The growth curves of Lactobacillus sp. reached a plateau phase after $24 \mathrm{~h}$ cultivation with FOS, whereas it took $36 \mathrm{~h}$ to reach the plateau phase without FOS. Therefore, the growth rate of the bacteria in FOS-containing medium was faster than that in the medium without FOS. Moreover, the nal bacterial mass in the medium with FOS was found to be greater than that in the medium without FOS. Furthermore, the $\mathrm{pH}$ values of the media dropped along with the increases of beneficial bacterial populations. The findings of our study agree with those of $\mathrm{Li}$ et al. (2008) where the $1 \%(\mathrm{w} / \mathrm{v})$ FOS had a stimulatory effect on the lactobacilli and bifidobacteria used. In addition, it was observed that as the growth rate increased, the $\mathrm{pH}$ decreased simultaneously.

During the fermentation of fructose oligomers by probiotic bacteria, authors have observed a gradual decrease in the $\mathrm{pH}$ which is indicative of the production of lactic acid (Rastall et al., 2000). The decrease in pH va- lues after incubation with FOS suggests that the bi dobacteria were able to utilize FOS. These results indicate that FOS potently stimulated the growth of bi dobacteria, which supported the potential prebiotic effect of FOS.

In this study, the FOS produced by $A$. pullulans was able to enhance the growth of prebiotic bacteria that are native to the gastrointestinal microbiota; therefore, the use of these FOS as food additives is advised.

\section{Conclusion}

In this study, we synthesized and evaluated the crosslinked fructosyltransferase aggregates. The conditions for the CLEA preparation were statistically optimized, a step necessary for a scale-up process for industrial/ biotechnological biocatalytic applications such as the mass production of the prebiotic FOSs. The structural characterization of the CLEAs revealed nonuniform morphological features while sulfur, oxygen, and phosphorus were established to be present in high amounts. Our report is the first to use a statistical approach for the optimization of fructosyltransferase CLEA production. Based on CLEA reusability, the fructosyltransferase CLEAs can be applied as industrial biocatalysts in the production of FOS. The produced FOS had prebiotic properties comparable to those obtained from a commercially obtained FOS, hence $A$. pullulans NAC8 FOSs could be a novel source for its production.

\section{Acknowledgements}

The authors wish to thank Dr. A. J. Akinloye of the Department of Botany, Obafemi Awolowo University, Ile-Ife, Mr. Tomide of the Center for Energy Research and Development (CERD), Obafemi Awolowo University, Ile-Ife and technologists in the Material Research Department of iThemba Labs, Cape Town, South Africa for the help with the microscopic analysis of the CLEA, Fourier Transform Infrared Spectroscopy and the scanning electron microscopy energy-dispersive X-ray analysis respectively.

\section{Disclosure}

All authors declare no conflict of interest.

\section{References}

Ademakinwa N.A., Agboola, F.K. (2016) Biochemical characterization of a novel laccase newly obtained from Aureobasidium pullulans NAC8 isolated from soil containing decayed plant litters. J. Genet. Eng. Biotechnol. 14(1): 346-356. 
Ademakinwa N.A., Ayinla Z.A., Agboola F.K. (2017) Strain improvement and statistical optimization as a combined strategy for improving the fructosyltransferase production by Aureobasidium pullulans NAC8. J. Genet. Eng. Biotechnol. 15(2): 345-358.

Ayhan H., Ayhan F., Gulsu A. (2012) Highly biocompatible enzyme aggregates cross-linked by L-lysine. Turk. J. Biochem. 37: 14-20.

Adina C., Fetea F., Taoutaou A., Carmen S (2010) Application of FTIR spectroscopy for a rapid determination of hydrolytic enzymes activity on sea Buckthron substrate. Rom. Biotech. Lett. 15(6): 5738-5744.

Bali V., Panesar P.S., Bera M.B., Panesar R. (2015) Fructooligosaccharides: Production, purification and potential applications. Crit. Rev. Food Sci. Nutr. 55(11): 1475-1490.

Broun G.D. (1976) Chemically aggregated enzymes. Meth. Enzymol. 44: 263-280.

Cao L., van Langen L., Sheldon R.A. (2003) Immobilised enzymes: Carrier-bound or carrier-free?Curr. Opin. Biotechnol. 14: 387-394.

Cao L., Van Rantwijk F., Sheldon R.A. (2000) Cross-linked enzyme aggregates: $A$ simple and effective method for the immobilization of penicillin acylase. Org. Lett. 2: 1361-1364.

Chien C.S., Lee W.C., Lin T.J. (2001) Immobilization of A. japonicus by entrapping cells in gluten for production of fructooligosaccharides. Enzyme Microb. Technol. 29: 252-257.

Duarte I.F. Barros A., Delgadillo I. (2002) Application of FTIRspectroscopy for the quantification of sugars in Mango Juice as a function of ripening. J. Agric. Food Chem. 50: 3104-3111.

Fernandes P. (2010) Enzymes in food processing: a condensed overview on strategies for better biocatalysts. Enzyme Res. 1: 19

Fernandez J.F.A., McAlpine M., Halling P.J. (2005) Operational stability of subtilisin CLECs in organic solvent in repeated batch and continuous operation. Biochem. Eng. J. 24: 11-15.

Fernandez-Fernandez M., Sanmoran M.A., Moldes D. (2013) Recent development and application of immobilized laccase. Biotechnol. Adv. 33: 404-418.

Flores-Maltos D.A., Mussatto S.I., Teixeira J.A. (2014) Biotechnological production and application of fructooligosaccharides. Crit. Rev. Biotechnol. 1(9): 1-9.

Ganaie A.M., Rawat K.H., Wani O.A., Gupta U.S., Kango N. (2014) Immobilization of fructosyltransferase by chitosan and alginate for efficient production of fructooligosaccharides. Process. Biochem. 49: 840-844.

Ghazi I., Gomez A., Arrojo L., Yates M., Cervantes L., Plou F.J. (2005) Immobilisation of fructosyltransferase from Aspergillus aculeatus on epoxy-activated Sepabeads EC for the synthesis of fructo-oligosaccharides. J. Mol. Cat. B: Enzymatic 35(1-3): 19-27.

Haaland P.D. (1989) Experimental design in biotechnology. Marcel Dekker Inc., New York. pp. 76-77.
Hayashi S., Ito K., Nonoguchi M., Takasaki Y., Imada K. (1991) Immobilization of a fructosyl-transferring enzyme from Aureobasidium sp. on shirasu porous glass. J. Biosci. Bioeng. 72(1): 68-70.

Jung C. (2000) Insight into protein structure and protein ligand recognition by Fourier Transform infrared spectroscopy. J. Mol. Recog. 13(6): 325-351.

Jung K.H., Bang S.H., Oh T.K., Park H.J. (2011) Industrial production of fructooligosaccharides by immobilized cells of Aureobasidium pullulans in a packed bed reactor. Biotech. Lett. 33: 1621-1624.

Li D., Kim J.M., Jin Z., Zhou J. (2008) Prebiotic effectiveness of inulin extracted from edible burdock. Anaerobe 14: 29-34.

Kaplan H., Hutkins R.W. (2000) Fermentation of fructooligosaccharides by lactic acid bacteria and bifidobacteria. Appl. Env. Microb. 66: 2682-2684.

Maiorano A.E., Silva E.S., Piccoli R.A., Ottoni C.A., Guilarte B., Cuervo R., Moreira R., Rodrigues M.F.D.A. (2009) Influence of the culture medium on the fructosyltransferase production. N. Biotechnol. 25(1): 201-207.

Mateo C., Palomo J.M., Fernandez-Lorente G., Guisan J.M., Fernandez-Lafuente R. (2007) Improvement of enzyme activity, stability and selectivity via immobilization techniques. Enzyme Microb. Tech. 40: 1451-1463.

Migneault I., Dartiguenave C., Bertrand M.J., Waldron K. (2004) Glutaraldehyde: behavior in aqueous solutions, reactions with proteins and applications to enzyme crosslinking. Biotechniques 37(5): 790-802.

Miller G.L. (1959) Use of dinitrosalicylic acid reagent for determination of reducing sugars. Anal. Chem. 31(3): 426-428.

Nobre C., Teixeira J.A., Rodrigues L.R. (2012) Fructooligosaccharides purification from a fermentative broth using an activated charcoal column. N. Biotechnol. 29(3): 395-401.

Ortiz-Soto M.E., Pinera E.R., Rodigrez-Algeria M.E., Munguia A.L. (2009) Evaluation of cross-linked enzyme aggregates from purified Bacillus subtilis levansucrase mutants for transfructosylation reactions. BMC Biotechnol. 9(68): 1-8.

Park H.J., Uhm K.N., Kim H.K. (2010) Biotransformation of amides to acids using a co-crosslinked enzyme aggregate of Rhodococcus erythropolis Amidase. J. Microbiol. Biotechnol. 20(2): 325-331.

Rastall R.A., Maitin V. (2002) Prebiotics and synbiotics: towards the next generation. Curr. Opin. Biotechnol. 13: 490-496.

Sabater-Molina M., Larque E., Torrella F., Zamora S. (2009) Dietary fructooligosaccharides and potential benefits on health. J. Physiol. Biochem. 65: 315-328.

Schoevaart R., Wolbers M.W., Golubovic M., Ottens M., Kieboom A.P.G., van Rantwijk K., Wielen L.A.M., Van der Ra S. (2004) Preparation, optimization and structures of cross-linked enzyme aggregates (CLEAs). Biotechnol. Bioeng. 87(6): 754-762.

Sheldon R.A. (2007) Enzyme immobilization: the quest for optimum performance. Adv. Syn. Catal. 349: 1289-1307. 
Susi H., Byler D.M. (1986) Resolution-enhanced Fourier transform infrared spectroscopy of enzyme. Meth. Enzymol. 130: 290-311.

Talekar S., Nadar S., Joshi A., Joshi G. (2014) Pectin crosslinked enzyme aggregates (Pectin-CLEA) of glucoamylase. Royal Soc. Chem. Adv. 4: 59444-59453.

Tanriseven A., Aslan Y. (2005) Immobilization of Pectinex UItra SP-L to produce fructooligosaccharides. Enzyme Microb. Technol. 36: 550-554.

Tischer W., Kasche V. (1999) Immobilized enzymes: Crystals or carriers? Trends Biotechnol. 17: 326-335.

Trollope K.M. Volschenk H., Gorgens J.F., Bro R., Nieuwoudt H.H. (2015) Direct and simultaneous quantification of fructooligosaccharides by FT-MIR-ATR spectroscopy for rapid identification of superior beta-fructofuranosidases. Anal. Bioanal. Chem. 407: 1661-1671.

Yun J., Song S. (1996) Continuous production of fructooligosaccharides using fructosyltransferase immobilized on ion exchange resin. Biotechnol. Bioprocess. Eng. 1: 18-21.
Wang A., Zhang F., Chen F., Wang M., Li H., Zeng Z., Xie T., Chen Z. (2011) A facile technique to prepare cross-linked enzyme aggregates using p-benzoquinone as cross-linking agents. Korean J. Chem. Eng. 28: 1090-1095.

Wang S., Zheng D., Yin L., Wang F. (2017) Preparation, activity and structure of cross-linked enzyme aggregates with nanoparticles. Enzyme Microbial. Technol. DOI: 10.1016. j.enzmictech.2017.07.008 (in press).

Wilson L., Illanes A., Abian O., Pessela B., Fernández-Lafuente R., Guisán J.M. (2004) Co-aggregation of penicillin $G$ acylase and polyionic polymers: a simple methodology to prepare enzyme biocatalysts stable in organic media. Biomacromolecules 5(3): 852-857. 